\title{
Informações médicas na internet afetam a relação médico-paciente?
}

Elisa Quaresma Coelho ${ }^{1}$, Augusto Quaresma Coelho ${ }^{2}$, José Eduardo Dias Cardoso ${ }^{3}$

\section{Resumo}

Este artigo pretende avaliar o impacto, sobre a relação médico-paciente, das informações disponíveis na internet. Foram aplicados questionários para 221 pacientes e 84 médicos, seguidos de análise descritiva dos dados, bem como incluídos pacientes com fácil acesso à internet e médicos que os atendem. A pesquisa, feita pelo paciente, muitas vezes resultou em pacientes melhor informados. Relevante número de pacientes se sentiu preocupado pela falsa impressão de portar males descritos na internet. Alguns recorreram à automedicação, modificação da prescrição médica ou, mesmo, à troca de profissional. Não é infrequente ocorrer prejuízo ao tratamento e à relação médico-paciente relacionado às informações obtidas e seu entendimento pelo paciente. Conclui-se que ao potencial "iatrogênico" da internet se contrapõe sua capacidade de difundir importantes informações à população. A certificação de sites por entidade reguladora, gerando qualidade de informação e menor "iatrogenia", associada à orientação, poderia ser útil para aperfeiçoar a relação médico-paciente. Palavras-chave: Internet. Bioética. Saúde. Informação. Ética. Relação médico-paciente.

\section{Resumen}

\section{¿Las informaciones médicas disponibles en la Internet pueden afectar la relación médico-paciente?}

Este artículo pretende evaluar el impacto de la información disponible en la Internet acerca de la relación médico-paciente. Cuestionarios fueron administrados a 221 pacientes y a 84 médicos, seguidos de análisis descriptivo de los datos. Los pacientes con fácil acceso a internet y los médicos que los atienden fueron incluidos en el estudio. La búsqueda realizada por el paciente, a menudo resultó pacientes mejor informados. Un relevante número de los pacientes se sintió preocupado por la falsa impresión de tener las enfermedades descritas en la Internet. Algunos recurrieron a la automedicación, la modificación de la prescripción médica o aun el cambio de los profesionales. No es infrecuente existir perjuicio al tratamiento y a la relación médico-paciente gracias a la información obtenida por el paciente y su comprensión de ella. A la conclusión se verifica que el potencial "iatrogénico" de la Internet se opone a su capacidad de difundir informaciones importantes a la población. La certificación de sitios web por institución reguladora, proporcionando mejor calidad de la información y reducción de "iatrogenia", asociada a la orientación, podría ser útil para perfeccionar la relación médico-paciente. Palabras-clave: Internet. Bioética. Salud. Información. Ética. Comunicación médico-paciente.

\section{Abstract \\ Does the information currently available on the Internet affect the physician-patient relationship?}

This article evaluates the impact of information available on the internet over the physician-patient relationship. The questionnaires were applied to 221 patients and 84 physicians followed by a descriptive data analysis. Patients with easy access to the internet and doctors who consult these people were included in the study. The research when done by the patient often resulted in better informed patients. A significant number of patients said they felt worried about having a false impression of having diseases described on the internet. Some have resorted to self-medication, modification of the prescription or even the exchange of the attending professional. Not infrequently there is damage to treatment and doctor-patient relationship due to information obtained by the patient and their understanding of them. At conclusion the "iatrogenic" potential of the internet is opposed to its ability to disseminate important information to the people. Certification of sites by regulatory authorities, improving information quality and reducing "iatrogenic", associated with the guidance of doctors, could be useful to improve the doctor-patient relationship.

Key words: Internet. Bioethics. Health. Information. Ethics. Physician-patient relationship.

Aprovação CEP CEPSH/Unilus 076 em 24/11/2010

1. Graduanda elisaqcoelho@hotmail.com - Centro Universitário Lusíada (Unilus), Santos/SP, Brasil 2. Graduando augustoqcoelho@ gmail.com - Universidade de São Paulo (USP), São Paulo/SP, Brasil 3. Especialista eduardocardoso@uol.com.br - Conselho Regional de Medicina do Estado de São Paulo (Cremesp), São Paulo/SP, Brasil.

\section{Correspondência}

Elisa Quaresma Coelho - Rua República Argentina, 12, apt 61 CEP 11065-030. São Paulo/SP, Brasil.

Declaram não haver conflito de interesse. 
A internet tem sido cada vez mais utilizada como fonte de pesquisa e meio de comunicação quando o assunto é saúde ${ }^{1}$, fato devido, principalmente, por ser instrumento de fácil acesso, conveniente e anônimo ${ }^{2,3}$. A democratização possibilitada pela internet ao acesso à informação constitui ganho fundamental para a sociedade e pode se tornar poderoso instrumento à prevenção de doenças e promoção da saúde ${ }^{4,5}$. Entretanto, para que isso ocorra, faz-se preciso considerar três questões: a qualidade das informações; a forma como são apresentadas ao público leigo e a seriedade dos autores do site ou o condicionamento das informações disponibilizadas aos interesses econômicos e pessoais do autor.

Estudo realizado pela Health On the Net Foundation (HON) em julho e agosto de $2010{ }^{1}$, com o intuito de verificar a tendência da postura do público em geral e de profissionais da área da saúde em relação ao uso da internet para fins de saúde desde 1996, constatou que, para a população em estudo (524 pessoas), a qualidade da informação continua a ser a principal barreira para a pesquisa. Dentre os fatores considerados para aumentar a qualidade das informações online sobre saúde, os mais valorizados foram confiabilidade/credibilidade; precisão; disponibilidade da informação e facilidade de busca de informações e navegação.

Este mesmo estudo ${ }^{1}$ mostra que tanto o público em geral quanto os profissionais de saúde que discutem com seus pacientes as buscas por eles feitas na internet relatam que o efeito é positivo: meIhora a comunicação e encoraja o paciente a lutar contra a sua doença. No entanto, de modo geral, os médicos acreditam que isto também possa aumentar o risco da automedicação, mais do que os próprios pacientes referem pensar no assunto ${ }^{1}$.

Os temas mais controversos no estudo feito pela fundação $\mathrm{HON}^{1}$ foram: 1) se a discussão sobre informações de saúde lidas na internet promove a desconfiança do paciente; 2) se encoraja os pacientes a desafiar a autoridade de um médico. Porém, outros estudos sugerem que o médico ainda é a fonte mais importante de informação sobre saúde e que os pacientes veem a internet como recurso adicional de apoio à relação médico-paciente, o que atuaria como uma tentativa, por parte do paciente, de trabalhar em conjunto com o médico ${ }^{2,3}$.

Estudos mostraram que a quantidade de informações duvidosas encontradas em diversos sites de saúde é enorme e que alguns deles contêm, inclusive, informações potencialmente enganosas ${ }^{1,6,9}$. Dada a capacidade de a internet rapidamente disseminar qualquer conteúdo, estes tra- balhos também advertem acerca da disseminação de informações falsas ou tendenciosas em saúde pela rede, que poderiam ser perigosas ou mesmo prejudiciais ao grande público ${ }^{7,8}$. Tal constatação tem gerado preocupação, pois existem bases sólidas para aferir o poder da comunicação online.

Diversos estudos com a proposta de avaliar os sites disponíveis na internet sobre temas específicos de saúde observaram a dificuldade enfrentada pelo paciente em encontrar páginas de qualidade para suprir suas necessidades de informação ${ }^{7,9,10-14}$. Os resultados da pesquisa da fundação HON ${ }^{1}$ demonstram que o público em geral está se tornando mais crítico e menos satisfeito com a qualidade das informações de saúde obtidas por meio da internet. A maioria dos entrevistados reconhece o problema da falta de credibilidade de muitas fontes de informação e acredita que consegue avaliar criticamente o que lê ${ }^{1}$. Apesar disso, essas questões não deixam de ser preocupantes, especialmente porque contêm o potencial de afetar negativamente a relação médico-paciente.

Se a literatura discute como controlar ou avaliar a qualidade da informação na rede, ainda não há nenhum acordo a respeito ${ }^{7,12,15}$. A ideia de implementar um programa de aprovação de sites é considerada inviável, considerando a grande quantidade de sites para cada assunto ${ }^{9}$. Segundo alguns autores, o público geral entenderia melhor um "selo de aprovação" dado por um indivíduo ou grupo comumente conhecido como confiável ${ }^{7,9,12}$. Outras soluções propostas são a utilização de folhetos informativos ${ }^{12}$, recomendação de sites adequados pelos profissionais de saúde ${ }^{11,12,15}$, ferramentas de filtragem de sites ${ }^{12}$ e regulamentação governamental ${ }^{7}$.

Segundo a pesquisa da $\mathrm{HON}^{1}$, $80 \%$ da população em estudo acredita que um profissional de saúde deveria sugerir fontes confiáveis de informação para que o paciente pesquise na internet. $A$ respeito, $72 \%$ dos profissionais concordam que seria útil fornecer tais informações a seus pacientes. A maioria dos médicos relatou que usaria um serviço confiável online que lhes permitisse sugerir sites a seus pacientes, especialmente se gratuito. No entanto, até agora, $78 \%$ dos pacientes relataram que os profissionais de saúde nunca lhes deram tal informação.

\section{Objetivo}

O presente trabalho objetiva avaliar o impacto que as informações sobre saúde, disponíveis na 
internet, exercem sobre a relação médico-paciente, bem como sobre a eficácia das medidas terapêuticas propostas e utilização dos recursos de saúde, tendo em vista a inexistência de regulamentação específica para a disponibilização de conteúdo sobre saúde na internet e para o comércio eletrônico no Brasil.

\section{Material e método}

O desenho de pesquisa buscou elaborar um estudo descritivo, observacional, transversal para análise quantitativa de frequências. Foram aplicados dois tipos de instrumentos - para pacientes e médicos - e ambos os questionários, bem como os termos de consentimento livre e esclarecido (TCLE), foram aprovados pelo Comitê de Ética em Pesquisa envolvendo Seres Humanos do Centro Universitário Lusíada (CEPSH/Unilus) em 24.11.2010.

\section{Questionário para pacientes}

$\mathrm{O}$ instrumento utilizado para o levantamento de dados dos pacientes foi um questionário com 29 questões de múltipla escolha e algumas abertas complementares, que tratavam da relação entre os pacientes e a internet - elaborado com base na nona e décima edições da HON survey of health and medical internet users ${ }^{16,17}$ e devidamente adequadas pelos autores.

As primeiras questões atêm-se à análise do perfil do paciente: sexo, idade, escolaridade e como procura as informações sobre assuntos em geral. A seguir, como este utiliza a internet: se busca informações sobre medicina, frequência de acesso, método de escolha dos sites pesquisados, que informação pesquisa, se compartilha as informações obtidas, se encontra dificuldade em identificar sites confiáveis, confiabilidade das informações contidas e grau de dificuldade para entender o conteúdo dos sites pesquisados.

Por fim, o questionário abordava as possíveis alterações de conduta após busca na internet: se já se automedicou; se já modificou sua postura perante o médico; se percebeu mudança de postura do médico após ser informado da pesquisa na internet; se voltou a conversar com o médico no caso de divergências entre o lido na rede e o por ele explicado; e se já se preocupou por pensar ser portador de doença cuja descrição encontrou na internet. A partir daí, as perguntas investigavam como foi essa preocupação (se comprometeu suas atividades diárias, se gerou ansiedade e/ou depressão) e se buscou esclarecimento com um profissional de saúde (em caso positivo, mais duas questões: quantos profissionais consultou para certificar-se de que estava ou não doente e se houve a necessidade de realizar exames adicionais para afastar esta preocupação).

\section{Aplicação do instrumento}

Após explanação sobre a finalidade do estudo, o questionário foi respondido pelo paciente ao pesquisador durante a espera por exame laboratorial, seguida de apresentação do termo de consentimento livre e esclarecido. A escolha dos pacientes foi aleatória, de acordo com sua apresentação na recepção do setor particular (separadamente do atendimento por convênios) do estabelecimento Instituto de Análises Clínicas de Santos, em Santos/ SP. Durante quatro semanas foram aplicados 221 questionários, no período da manhã, de dezembro de 2010 a janeiro de 2011.

\section{Questionário para médicos}

O questionário para os médicos continha 19 questões de múltipla escolha e algumas abertas complementares, que tratavam da relação entre os médicos e a internet e, também, entre os médicos e os pacientes que utilizam a rede. Este questionário também foi elaborado com base na nona e décima edições da HON Survey on Health and Medical Internet Use ${ }^{16,17}$, porém bastante modificado por iniciativa dos autores, buscando melhor adequá-lo ao escopo do estudo.

As primeiras questões voltavam-se à análise do perfil do médico: sexo, idade, tempo de exercício da profissão e especialidade. A estas, outras indagavam sobre o uso da internet: se busca informações sobre medicina na rede, qual a frequência de acesso e se participa da elaboração de sites relacionados à saúde. Algumas questões estavam direcionadas ao conhecimento do médico sobre o uso da internet pelos pacientes: se costuma verificar as informações às quais a população tem acesso, qual a frequência com que seus pacientes se apresentam à consulta informados sobre sua enfermidade e/ou tratamento tendo como fonte pesquisas na internet, se há preocupação com a origem dessas informações e se acredita que seus pacientes tenham dificuldade em achar informações que esclareçam suas dúvidas.

Outras perguntas abordavam as possíveis alterações de conduta após o paciente ter feito a busca na internet: se é frequente a necessidade de orientá-lo devido ao mau entendimento, se tem conhecimento de que o paciente se automedicou após consultar as informações, se algum paciente 
modificou alguma prescrição ou orientação com base nessas informações, se o paciente modificou sua postura, perante o médico, após a pesquisa na internet, e se o próprio médico modificou sua postura ante tal situação. Por fim, o questionário investigava a interferência na relação médico-paciente da utilização da internet pelo paciente: o grau de incômodo do médico ao ser confrontado com dados que o paciente pesquisou antes ou após a consulta, se em decorrência de informações obtidas na internet houve prejuízo ao tratamento proposto e se houve dificuldade na relação com o paciente por conta de divergências originadas por pesquisa na rede.

\section{Aplicação do instrumento}

A aplicação do instrumento para os médicos foi feita, basicamente, mediante visitas em consultórios particulares. Uma carta foi deixada para cada profissional, recolhida após uma semana. A escolha dos consultórios foi aleatória: oito prédios comerciais na cidade de Santos/SP. Nestes, foram percorridos todos os andares e as cartas entregues nos consultórios onde havia médicos, de qualquer especialidade. Foram distribuídos 87 questionários durante o período de dezembro de 2010 a janeiro de 2011 - três dos quais excluídos por preenchimento incorreto, perfazendo 84 instrumentos efetivamente aplicados.

\section{Resultados}

A amostra de pacientes, contendo 221 pessoas, foi em sua maioria composta por mulheres $(63,35 \%)$, das quais $91,4 \%$ com ensino médio completo, $50,23 \%$ com ensino superior completo e 5,43\% com pós-graduação. A maioria era jovem, entre 20 e 29 anos (25,34\%). A população feminina da amostra se dividia de forma semelhante no intervalo entre as décadas de 30 a 60, com média de $15,5 \%$ por década. Por sua vez, o perfil dos médicos apresentou maioria de homens $(60,71 \%)$, com $29,76 \%$ na faixa de 50 a 59 anos; $19,05 \%$, de 40 a 49 anos; $17,86 \%$, de 30 a 39 anos e $19,05 \%$, de 60 a 69 anos. Dos 84 médicos efetivamente participantes, $28,58 \%$ tinham de 0 a 10 anos de profissão e $40,48 \%$, de 25 a 35 .

Dos 221 pacientes, 163 (73,75\%) buscam informações sobre medicina pela internet e, destes, $56,11 \%$ acessam a rede diariamente; $80,98 \%$ procuram a descrição da doença; 50,31\% o tratamento e, em menor escala, também por casos semelhantes (30,06\%). Ainda, 26,99\% consultam artigos médi- cos, informações sobre prevenção, notícias e bulas. Como método de escolha dos sites, 95,71\% utilizam sites de busca ou sites aleatórios; 32,52\% recebem indicação de profissionais de saúde; 30,67\% recebem indicação de amigos e 19,63\% procuram em sites de indústrias farmacêuticas.

Concentrando a análise nos 163 pacientes que pesquisam informações médicas na internet, constatou-se que 58,28\% costumam dividir as informações lidas com seu médico; 87,7\%, com parentes e amigos e 6,13\% não as divulgam a ninguém. Quanto à confiança nos conteúdos pesquisados, 17,79\% sempre acreditam nas informações encontradas; $54,6 \%$ informam ter dificuldade em achar sites confiáveis e 32,52\% relatam sempre ter o cuidado de escolher sites ligados a instituições registradas no Conselho Regional de Medicina do Estado de São Paulo (Cremesp).

Dos 163 pacientes entrevistados, 56 (34,36\%) relataram nunca ter comentado com o médico sobre a busca feita e que também não modificaram a postura mesmo após a pesquisa na internet. No entanto, 20,24\% perceberam que o médico modificou a postura após a pesquisa (Gráfico 1); 12,27\% relataram que o médico explicou mais e/ou orientou melhor; $1,23 \%$ explicou que o médico modificou sua conduta e 4,29\% repararam que o médico "não gostou". Adicionalmente, $28,83 \%$ informaram ter modificado a postura perante o médico ou ao tratamento após a pesquisa (Gráfico 2), dos quais 17,79\% relataram que se sentiram mais capazes de questionar e entender, e 1,84\% mudou de médico.

Ainda com pertinência a estes pacientes, 72 $(44,17 \%)$ já se sentiram preocupados com a impressão de serem portadores de doença cuja descrição encontraram na internet. Em uma escala de 0 a 10, considerando 0 como "nenhuma preocupação" e 10 como "extrema preocupação", 38,9\% classificariam sua preocupação com nota de 8 a 10 e 45,8\%, com nota de 5 a 7 . No cômputo, 52,78\% relataram sentir ansiedade ou depressão em função desta preocupação; $20,8 \%$ relataram que isto comprometeu suas atividades diárias e 81,94\% buscaram esclarecimento com um profissional de saúde e $26,39 \%$ a mais de um médico.

Considerando 0 como nenhuma dificuldade e 10 como extrema dificuldade, os pacientes pesquisados foram questionados "se alguma vez sentiram dificuldade em entender o conteúdo apresentado pelo site pesquisado". Essa dificuldade foi classificada por 26,99\% como de 7 a $9 ; 29,45 \%$, de 4 a 6 e $30,06 \%$, de 1 a 3, e o restante relatou não sentir dificuldade alguma. Dentre as repercussões da busca 
feita pelos pacientes, questionamos a automedicação (Gráfico 3) e a iniciativa de modificar orientações ou prescrições médicas, admitidas por $21,47 \%$ e $9,2 \%$, respectivamente, e $3,68 \%$ referiram se automedicar com frequência.

Também foi indagado aos pacientes "se alguma vez pesquisaram na internet com a finalidade de confirmar se o tratamento ou o diagnóstico do médico que os consultou estavam corretos", ao que 75 deles responderam afirmativamente, representando 46,01\%. Destes, 54 (72\%) relataram divergência entre as informações lidas e as explicações do médico, e 46,29\% voltaram a discutir as informações com o profissional.

Mediante o questionário aplicado aos 84 médicos em estudo, foram obtidos os seguintes resultados para a pergunta sobre o acesso à internet no que tange às informações clínicas: $75 \%$ relataram acessar a internet diariamente; $88,1 \%$ buscam informações sobre medicina na rede; $21,43 \%$ são responsáveis ou participam de site relacionado à saúde; $41,67 \%$ costumam verificar o conteúdo direcionado à população leiga; 26,19\% vivenciaram dificuldades no relacionamento com pacientes por conta de divergências originadas por pesquisa na internet; 33,33\% acreditam que seus pacientes têm dificuldade em achar informações que esclareçam suas dúvidas; $27,38 \%$ narraram caso de prejuízo ao tratamento proposto devido à informação obtida pelo paciente na internet e $22,62 \%$ relataram que isto se devia a informações mal entendidas.

Em resposta ao questionário, $67,85 \%$ dos médicos comunicaram ser frequente ou muito frequente que seus pacientes já iniciem a consulta informados sobre a doença que apresentam (ou que pensam apresentar) ou sobre o tratamento proposto, tendo como fonte pesquisas na internet. Destes, $19,05 \%$ consideram essa situação muito frequente; $65,48 \%$ revelaram preocupar-se quase sempre com a origem das informações trazidas pelo paciente e $21,43 \%$ relataram preocupar-se, porém, nem sempre. Quanto ao pouco entendimento do paciente no tocante ao conteúdo pesquisado, que implica na necessidade de orientá-lo, os participantes consideram que é bastante frequente, sendo relatado por 91,67\% dos médicos.

Nos 84 questionários para médicos foi possível observar que a relação médico-paciente pode ser afetada pela pesquisa na internet pelo paciente. Verificou-se que $60,71 \%$ dos médicos têm conhecimento de que seus pacientes se automedicam a partir de informações da internet (Gráfico 3) e 28,57\% consideram este fato muito frequente. $42,85 \%$ depararam-se com históricos de pacientes que tomaram a iniciativa de modificar alguma prescrição ou orientação baseados em informações obtidas na internet.

Em 57,14\% das respostas, o médico sentiu que o paciente modificou a postura perante suas atitudes ou ao tratamento após pesquisar na internet, ficando mais atento ou mais duvidoso em relação às suas orientações (Gráfico 2), e 10,71\% admitiram que alguma vez modificaram sua postura perante 0 paciente após serem informados sobre a pesquisa na internet (Gráfico 1). Foi também questionado como o médico se sentia, de maneira geral, quando confrontado com dados que o paciente pesquisou antes ou após a consulta, considerando 0 como nenhum incômodo e 10 como extremo incômodo, obtendo-se os seguintes resultados: $4,76 \%$ sentem-se extremamente incomodados; $10,71 \%$ classificariam seu incômodo com nota entre 7 a 9; 35,71\% o avaliaram em 4 a 6; 26,19\% em 1 a 3 e 22,62\% negaram qualquer incômodo nesta situação.

Utilizamos o teste do qui-quadrado para verificar associações entre as variáveis sexo, idade e grau de instrução, no caso dos pacientes, com outras perguntas do questionário e idade, sexo e tempo de profissão, no caso dos médicos. O teste demonstra diferença estatística entre os grupos para algumas variáveis. No entanto, nem sempre é possível inferir se esta diferença é progressiva, maior ou menor, em relação aos dados estudados, dada a complexidade do questionário. Na questão dirigida aos pacientes, "se alguma vez pesquisaram na internet com a finalidade de confirmar se o tratamento ou o diagnóstico do médico que o consultou estavam corretos", foram apontadas diferenças estatísticas nos questionários associados às variáveis idade $(p=0,019)$ e grau de instrução $(p=0,023)$.

Para os pacientes, foi numericamente demonstrada associação de diferença entre a busca de informações sobre medicina pela internet com as variáveis idade, grau de instrução e sexo, mostrando que mulheres $(p=0,02)$, pessoas mais jovens $(p=0,003)$ e os mais graduados $(p=0)$ são os grupos que mais buscam esclarecer suas dúvidas sobre saúde por intermédio da internet. Com relação à dificuldade em achar sites confiáveis, constatou-se estar relacionada a pacientes de menos idade $(p=0,017)$. Percebeuse, ainda, que os homens são menos afetados pela impressão de ser portadores de doença cuja descrição encontraram na internet $(p=0,046)$. Obteve-se associação de diferença significativa também no questionário para os médicos no tocante à questão "sentiu que o paciente modificou a postura perante 
suas atitudes ou ao tratamento após pesquisar na internet (exemplo: ficou mais atento às suas orientações ou mais duvidoso em relação a elas)?", com maior frequência para os médicos menos experientes $(p=0,02)$ e com menos idade $(p=0,002)$.

Gráfico 1. Resposta de médicos e pacientes às perguntas: "Alguma vez modificou sua postura perante o paciente após ser informado sobre pesquisa na internet?"; "O médico modificou a postura perante você após sua pesquisa na internet?"

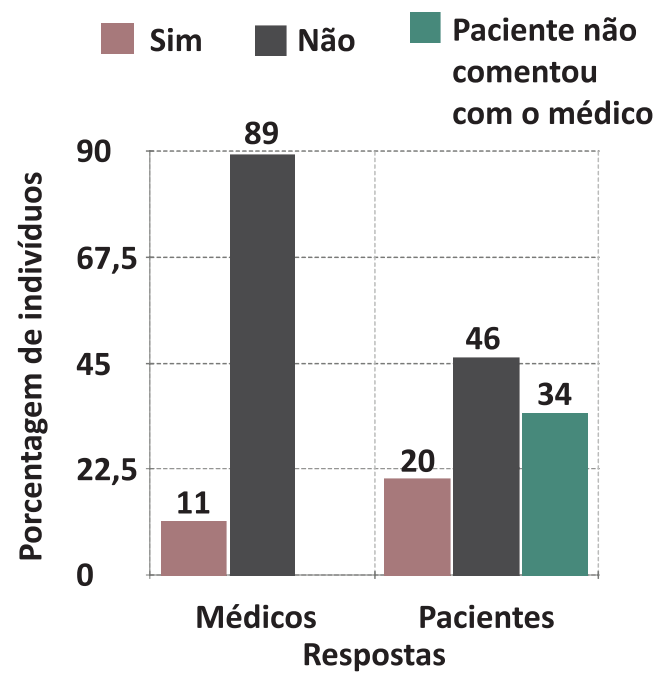

Gráfico 2. Resposta de médicos e pacientes às perguntas: "Sentiu que o paciente modificou a postura perante suas atitudes ou ao tratamento após pesquisar na internet (exemplo: ficou mais atento às suas orientações ou mais duvidoso em relação a elas)?"; "Modificou sua postura perante o médico ou ao tratamento após pesquisar na internet?"

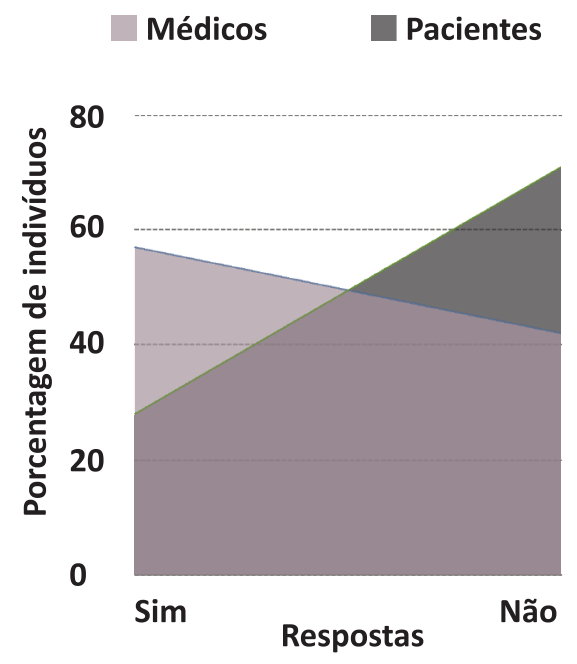

Gráfico 3. Resposta de médicos e pacientes às perguntas: "Já ocorreu de um paciente tomar a iniciativa de modificar alguma prescrição ou orientação sua baseada em informações obtidas na internet?"; "Já tomou a iniciativa de modificar alguma prescrição ou orientação médica feita a você após pesquisar o assunto na internet?"

Sim, mas é raro

Sim, não é raro

Não

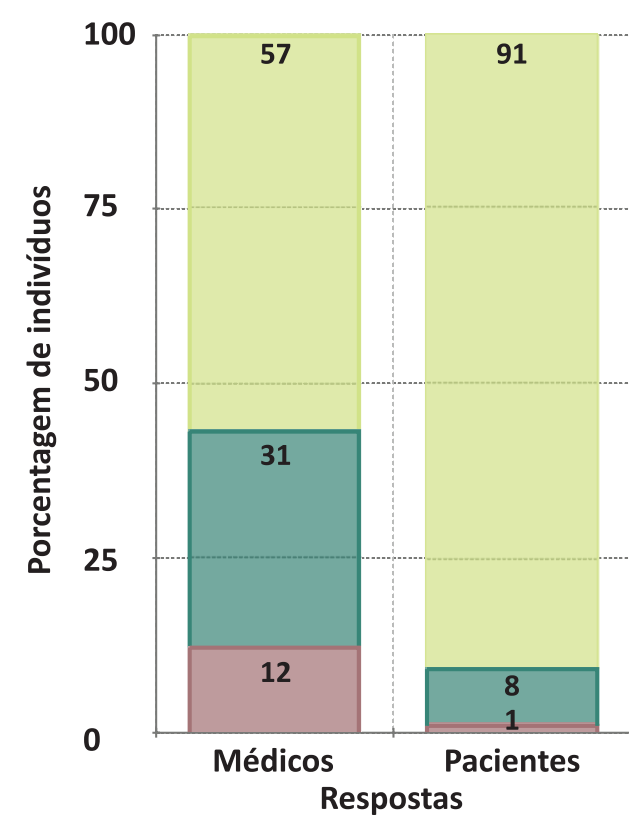

\section{Discussão}

Os dados encontrados parecem mostrar que a população estudada, apesar da elevada escolaridade, nem sempre seleciona adequadamente os sites que utiliza em suas pesquisas, sente dificuldade em achar sites confiáveis ou, ainda, em entender o conteúdo da pesquisa. No entanto, importante contingente acredita sempre no que lê e muitas vezes não discute com o médico os dados encontrados na internet.

É frequente que o paciente se apresente à consulta já tendo pesquisado sobre a doença. Tanto o médico quanto o paciente parecem mudar sua postura na relação quando há pesquisa feita pelo paciente. Em expressivo número de casos isso parece resultar em pacientes melhor informados e mais capazes de discutir com o médico, o que eventualmente pode refletir em melhores resultados no tratamento. $95(58,28 \%)$ pacientes afirmaram que cos- 
tumam dividir as informações lidas sobre saúde na internet com seu médico. Significativo contingente $(44,17 \%)$, entretanto, experimentou efeitos negativos decorrentes de sua pesquisa na internet.

Quase metade dos entrevistados já se sentiu preocupada pela falsa impressão de ser portadora de males descritos na internet, ao ponto de muitos se sentirem ansiosos ou deprimidos e/ou terem a sua rotina diária comprometida. Essa falsa preocupação levou à realização de consultas médicas, às vezes múltiplas, e exames subsidiários - a princípio desnecessários.

Após o diagnóstico, os sujeitos entrevistados pesquisam para se informar sobre sua doença e avaliar a conduta adotada pelo profissional. Houve caso, inclusive, de o paciente pesquisar na internet para confirmar se o tratamento e/ou diagnóstico do médico que o consultou estavam corretos. Número não desprezível de pacientes pesquisados já recorreu à automedicação, à modificação da prescrição médica ou, mesmo, à troca de profissional em razão de informações obtidas na internet.

A partir do relato dos médicos estudados, percebe-se não ser incomum a ocorrência de prejuízos ao tratamento e à relação médico-paciente relacionadas às informações obtidas na internet $\mathrm{e}$ seu entendimento pelo paciente. Muitos profissionais relataram sentir-se desconfortáveis diante do confronto de sua conduta com informações obtidas na internet - ou mesmo preocupados com sua origem. Aparentemente, os pacientes têm dificuldade em identificar sites que sejam confiáveis e que esclareçam suas dúvidas. Tanto as perguntas dirigidas a médicos como aos próprios pacientes apontam nesse sentido.

\section{Considerações finais}

O impacto na relação médico-paciente das informações em saúde disponíveis na internet, tanto sobre a eficácia das medidas terapêuticas quanto sobre a utilização dos recursos de saúde disponíveis, exige que a discussão acerca desse tema receba maior atenção. Ao potencial "iatrogênico" da internet se contrapõe a sua capacidade de difundir informações que facilitem a prevenção e o tratamento das doenças, contribuindo, assim, para a saúde da população. Para minimizar o primeiro aspecto e maximizar o segundo, faz-se preciso maior atenção ao conteúdo dos sites com informações sobre saúde.

Não cabe imaginar medidas restritivas ou de censura à internet, mas buscar a democratização da informação que torne a relação médico-paciente mais transparente e produtiva. Entretanto, é crível imaginar que sites certificados por entidade reguladora ofereçam melhor qualidade de informação, resultando em mais benefícios e menor "iatrogenia". Estudos poderiam ser desenhados para confirmar ou negar essa proposição. Assim, caso confirmada, a certificação voluntária e a orientação à população, para que utilize preferencialmente tais sites, poderiam ser instrumentos úteis para promover a saúde e aperfeiçoar a relação médico-paciente.

Outra sugestão, por fim, seria que os próprios médicos, em associações orientadas por especialidades, elaborassem listas de sites com informações confiáveis, contribuindo, assim, para a disseminação de conteúdos fidedignos em sua especialidade e para fortalecer a autonomia do paciente em relação à sua saúde e qualidade de vida.

\section{Referências}

1. Pletneva N, Cruchet S, Simonet M, Kajiwara M, Boyer C. Results of the $10^{\text {th }} \mathrm{HON}$ survey on health and medical Internet use. Stud Health Technol Inform. 2011;169:73-7.

2. Stevenson FA, Kerr C, Murray E, Nazareth I. Information from the internet and the doctor-patient relationship: the patient perspective, a qualitative study. BMC Fam Pract. 2007;8:47.

3. McMullan M. Patients using the internet to obtain health information: how this affects the patient-health professional relationship. Patient Educ Couns. 2006;63:24-8.

4. Silva W. Navegar é preciso: avaliação de impactos do uso da internet na relação médico-paciente. [dissertação]. São Paulo: Universidade de São Paulo; 2006.

5. Rapparini C, Barroso PF, Saraceni V, Machado AA, Fernandes GC. Occupationally acquired infectious diseases among health care workers in Brazil: use of internet tools to improve management, prevention, and surveillance. Am J Infect Control. 2007; 35(4):267-70.

6. Eysenbach G, Köhler C. Does the internet harm health? Database of adverse events related to the internet has been set up. BMJ. 2002;324(7331):239.

7. López-Jornet $P$, Camacho-Alonso $F$. The quality of internet information relating to oral leukoplakia. Med Oral Patol Oral Cir Bucal. 2010;15(5):727-31.

8. Jadad AR, Gagliardi A. Rating health information on the internet navigating to knowledge or to babel? Jama. 1998;279(8):611-4. 
9. Pérez-López FR. An evaluation of the contents and quality of menopause information on the world wide web. Maturitas. 2004;49(4):276-82.

10. Ellis $\mathrm{MJH}$, Thomson CE. Consumer health information on the www: an evaluation of information on verrucae. The Foot. 2003;13:130-5.

11. López-Jornet $P$, Camacho-Alonso F. The quality of patient-orientated Internet information on oral lichen planus: a pilot study. J Eval Clin Pract. 2010;16(5):883-6.

12. Rajani R, Mukherjee $D$, Chambers J. Murmurs: how reliable is information on the internet? Int $J$ Cardiol. 2007;119(1):112-3.

13. Tan $\mathrm{BH}$, Kostapanagiotou K, Jilaihawi AN. A review of mesothelioma information on the world wide web. J Thorac Oncol. 2009;4(1):102-4.

14. Jetha A, Faulkner G, Gorczynski P, Arbour-Nicitopoulos K, Martin Ginis KA. Physical activity and individuals with spinal cord injury: accuracy and quality of information on the Internet. Disabil Health J. 2011;4(2):112-20.

15. Lewiecki EM, Rudolph LA, Kiebzak GM, Chavez JR, Thorpe BM. Assessment of osteoporosiswebsite quality. Osteoporos Int. 2006;17(5):741-52

16. Health On the Net Foundation. Analysis of $9^{\text {th }} \mathrm{HON}$ survey of health and medical internet users: winter 2004-2005. [Internet]. Geneva: HON Foundation; 2006 [acesso 12 set. 2012]. Disponível: http://www.hon.ch/Survey/Survey2005/res.html

17. Health On the Net Foundation. Evolution of internet use for health purposes. [Internet]. Geneva: HON foundation; 2006 [acesso 12 set. 2012]. Disponível: http://services.hon.ch/cgibin/Survey/ Survey2010/quest_oct.pl

\section{Participação dos autores}

José Eduardo Dias Cardoso orientou a análise dos resultados e a elaboração do texto final. Elisa Quaresma Coelho aplicou os questionários, tabulou e analisou os dados, discutiu os resultados e elaborou o texto final. Augusto Quaresma Coelho contribuiu na análise estatística dos dados e discussão dos resultados.

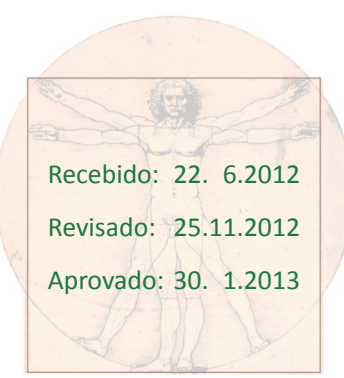

